\title{
Insights on molecular cloud structure
}

\author{
João Alves ${ }^{1}$, Marco Lombardi ${ }^{2,3}$, Charles Lada ${ }^{4}$ \\ ${ }^{1}$ University of Vienna, Türkenschanzstrasse 17, 1180 Vienna, Austria \\ ${ }^{2}$ ESO, Karl-Schwarzschild-Strasse 2, D-85748 Garching bei München, Germany \\ ${ }^{3}$ University of Milan, Department of Physics, via Celoria 16, I-20133 Milan, Italy (on leave) \\ ${ }^{4}$ Harvard-Smithsonian Center for Astrophysics, MS42, 60 Garden St., Cambridge, MA 02138 \\ email: joao.alves@univie.ac.at
}

\begin{abstract}
Stars form in the densest regions of clouds of cold molecular hydrogen. Measuring structure in these clouds is far from trivial as $99 \%$ of the mass of a molecular cloud is inaccessible to direct observation. Over the last decade we have been developing an alternative, more robust density tracer technique based on dust extinction measurements towards background starlight. The new technique does not suffer from the complications plaguing the more conventional molecular line and dust emission techniques, and when used with these can provide unique views on cloud chemistry and dust grain properties in molecular clouds. In this brief communication we summarize the main results achieved so far using this technique.
\end{abstract}

Keywords. ISM: clouds, dust, extinction, structure, stars: formation

\section{Introduction}

Stars are the outcome of the collapse of cold molecular clouds and yet we know much more about stellar structure than we know cloud structure. This is not surprising giving that clouds are not only the coldest known objects in the Universe but are made of molecular hydrogen $\left(\mathrm{H}_{2}\right)$ and helium both inaccessible to direct observation at the typical temperatures in these clouds $(\sim 10 \mathrm{~K})$. Instead, observers have been deriving the basic physical properties of these objects from observations of $\mathrm{H}_{2}$ trace molecules such as $\mathrm{CO}$ and $\mathrm{NH}_{3}$, as well as dust emission. Unfortunately, decoding observations of trace molecules or dust emission is not a straightforward task and several complications plague the derivation of basic cloud physical properties. An alternative way to derive cloud properties, and in particular cloud density structure, is to map the dust column density in these clouds, as dust is a robust surrogate of the molecular hydrogen. We have been developing a technique that makes use of reddened starlight seen through these clouds $†$. This technique produces column density maps that are more reliable and have a larger dynamic range than either dust emission or molecular line emission maps. Because dust extinction is such a robust tracer of mass (Goodman et al. 2009), the correlation between dust extinction and molecular line/dust emission maps provides unique views on the chemistry and dust grain properties in these clouds.

\section{Large Scale Extinction Maps}

With the availability of large near-infrared surveys, in particular the all-sky 2MASS survey, it became possible to apply the NICEST technique to essentially the entire sky. We took a systematic approach to produce the highest resolution maps possible of the most nearby cloud complexes, where confusion along the line-of-sight is minimal and

$\dagger$ The technique, first introduced by Lada et al. (1994), has had three improved versions: NICE (Alves et al. 1998), NICER (Lombardi \& Alves 2001), and NICEST (Lombardi 2009) 


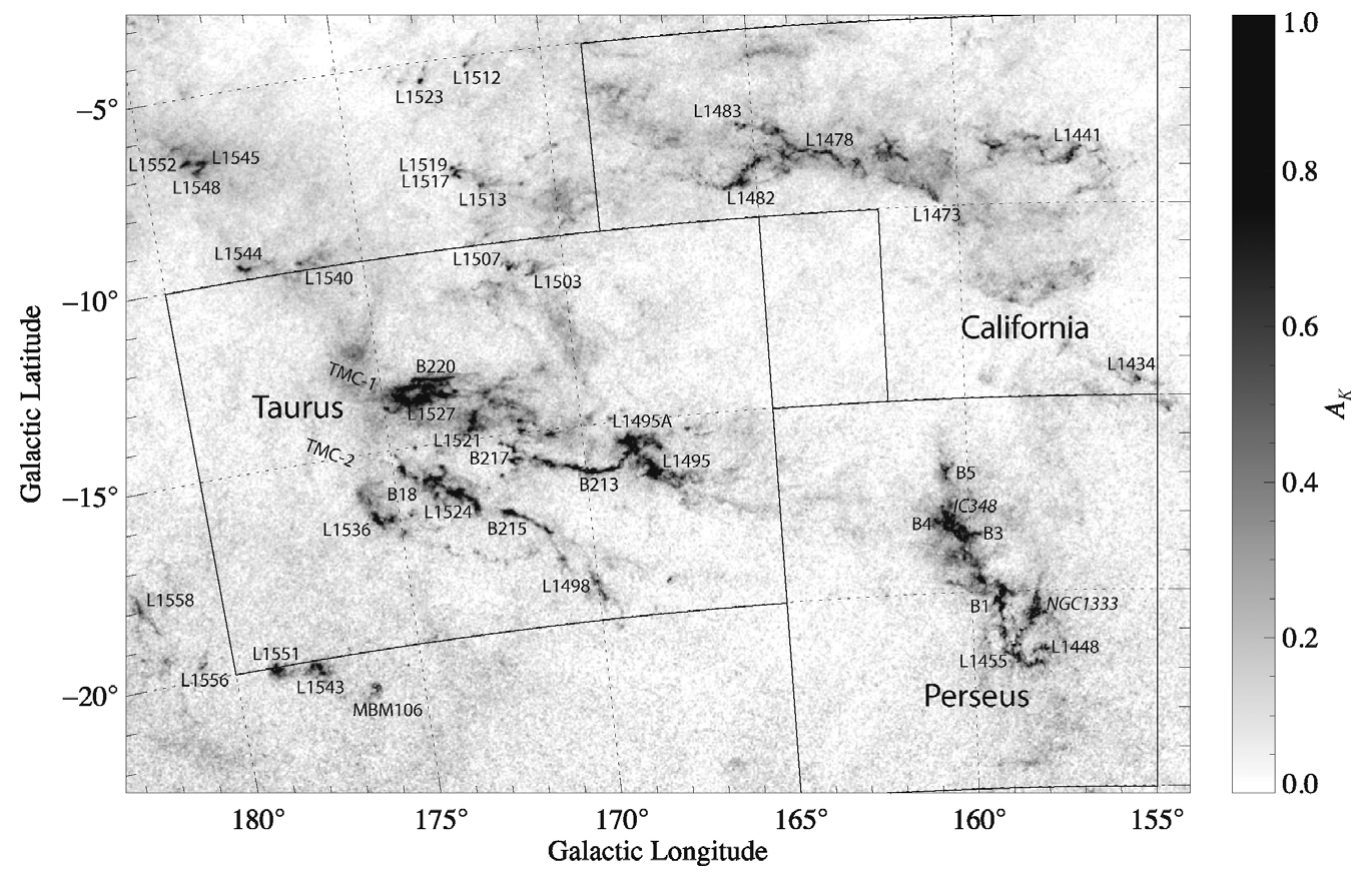

Figure 1. The NICER extinction map of the Taurus, Perseus, California complexes made from millions of background 2MASS stars (Lombardi et al. 2010b). The spatial resolution of the map is 2.5 arcmin.

distances are fairly well known (essentially objects belonging to the Gould's belt, e.g., Pipe Nebula, Ophiuchus, Lupus, Taurus, Perseus, California, and Orion). These maps (see Figure 1 for an example) provide an extraordinary view on these objects, from their largest, tens of parsec scales down to scales of about 2000 AU (e.g. Román-Zúñiga et al. 2010), and with unprecedented dynamic ranges of $10^{21}<\mathrm{N}<10^{23}\left(\mathrm{~cm}^{-2}\right)$. One of the main results from these large-scale maps of nearby complexes is that the same cloud description seems to be emerging repeatedly: all the nearby complexes are (large) filaments, with aspect ratios of the order of 10-100:1. At close inspection these filaments seem to be made of entanglements of narrower filaments (the streamers of Ophiuchus being the best example) loosely suggesting a self-similar organization. Apart a mass scale, the largest complexes (e.g., Orion) seem to have the same structure as the smaller complexes (e.g., Pipe). Another important cloud structure result is that about $90 \%$ of the mass of the nearby clouds lies at column densities below $\mathrm{A}_{V} \sim 5 \mathrm{mag}$, having little chance of ending up in the young stars forming in these clouds.

\section{The Universality of Molecular Cloud Structure}

An important result arising from the large-scale extinction maps is that different clouds have almost identical average column densities above a given extinction threshold (this statement holds regardless of the extinction threshold), indicating that molecular clouds are characterized by a universal structure (Lombardi et al. 2010a), a stringent confirmation of Larson's $3^{\text {rd }}$ law. An apparently independent result that has become obvious from dust extinction maps is that the column density distribution in these clouds can be well fitted by log-normal distributions, e.g., Lombardi et al. (2008); Kainulainen et al. (2009); Froebrich \& Rowles (2010). While it is not clear yet what is the ultimate relevance 


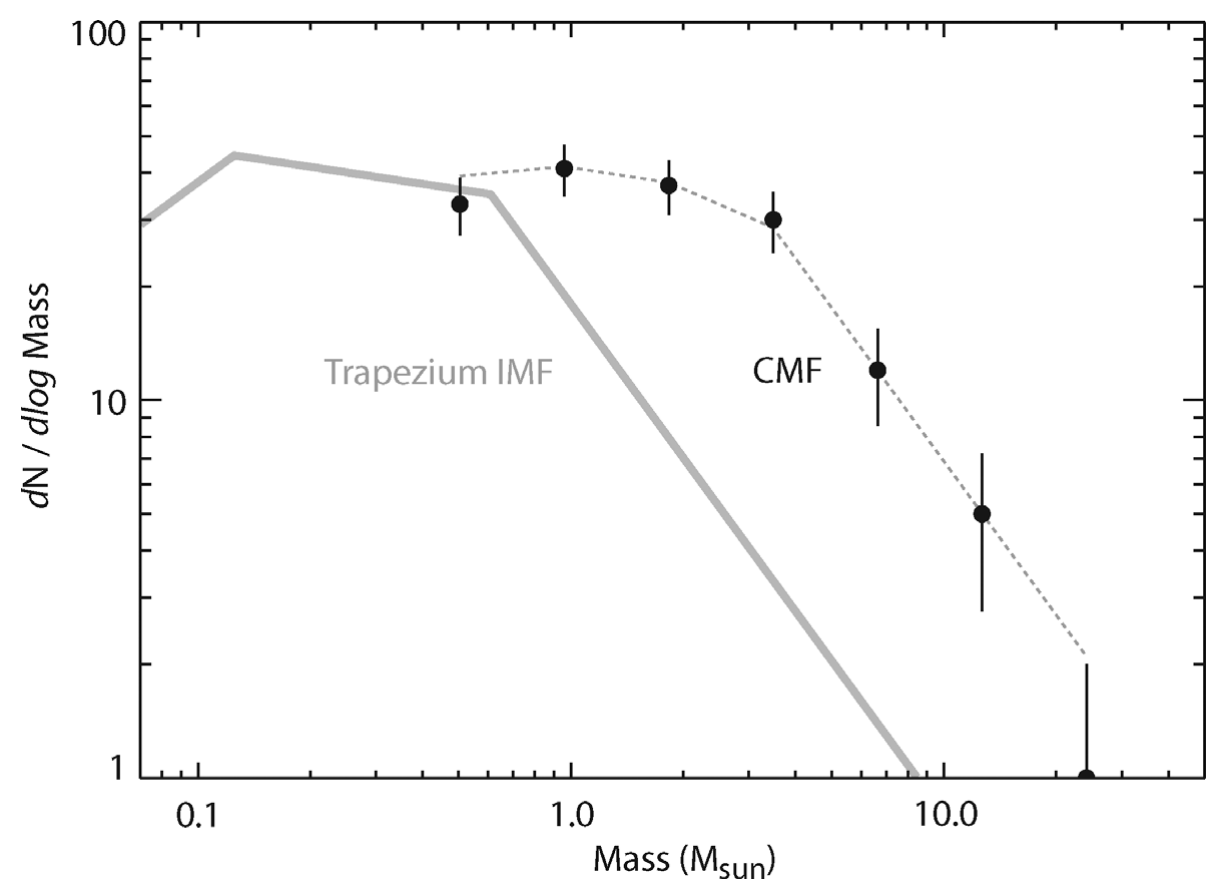

Figure 2. Mass function of dense molecular cores for the Pipe Nebula plotted as filled circles with error bars (Alves et al. 2007). The grey line is the stellar IMF for the Trapezium cluster (Muench et al. 2002). The dashed grey line represents the stellar IMF in binned form matching the resolution of the data and shifted to higher masses by about a factor of 4 . The dense core mass function is similar in shape to the stellar IMF function, apart from a (uniform) star formation efficiency factor $(\sim 30 \%)$.

of this result (Goodman et al. 2009; Tassis et al. 2010), we showed that the universal structure of these clouds can be linked to the log-normal nature of cloud column density distributions.

\section{Mass Distribution and the Star Formation Rate (SFR)}

Despite having the same overall structure, the fraction of the mass of a cloud at high column density varies immensely from cloud to cloud. For example, the California Nebula molecular cloud (Lada et al. 2009), while rivaling the Orion A molecular cloud as the largest and most massive GMC in the solar neighborhood, displays significantly less star formation activity with more than an order of magnitude fewer young stellar objects than found in the OMC. Analysis of extinction maps of both clouds shows that the California cloud contains only $10 \%$ the amount of high extinction $\left(\mathrm{A}_{K}>1.0 \mathrm{mag}\right)$ material as is found in the OMC, suggesting that the level of star formation activity and perhaps the star formation rate in these two clouds may be directly proportional to the total amount of high extinction material. Recently, Lada et al. (2010) found that the star formation rate (SFR) in molecular clouds is linearly proportional to the cloud mass $\left(\mathrm{M}_{0.8}\right)$ above an extinction threshold of $\mathrm{A}_{K} \approx 0.8$ magnitudes, corresponding to a gas surface density threshold of $\Sigma_{g a s} \approx 116 \mathrm{M}_{\odot} \mathrm{pc}^{2}$ corresponding to a volume density threshold of $\mathrm{n}\left(\mathrm{H}_{2}\right) \approx 10^{4} \mathrm{~cm}^{3}\left[\mathrm{SFR}\left(\mathrm{M}_{\odot} \mathrm{yr}^{1}\right)=4.6 \pm 2.6 \times 10^{8} \mathrm{M}_{0.8}\left(\mathrm{M}_{\odot}\right)\right]$. This relation between the rate of star formation and the amount of dense gas in molecular clouds appears to be in excellent agreement with previous observations of both galactic and extragalactic star forming activity (e.g. Wu et al. 2005). 


\section{The Core Mass Function and cores as Bonnor-Ebert spheres}

Moving to the small scales one finds a population of well defined extinction peaks with high column density. These column density peaks compare well in size and density with the dense ammonia cores first discussed in Myers \& Benson (1983), and appear to have, at least in the Pipe Nebula, a spatial distribution similar to the stars in Taurus, suggesting that the spatial distribution of stars evolves directly from the primordial spatial distribution of high density peaks (Román-Zúñiga et al. 2010). The structure of these dense cores, in particular the higher density ones, is well described by the equations for a pressure-confined, self-gravitating isothermal sphere that is critically stable according to the Bonnor-Ebert criteria (Alves et al. 2001). Using wavelet techniques to extract these cores we constructed a mass spectrum of high density peaks, or dense cores, and found evidence for a departure from a single power-law form in the mass function of a population of cores and find that this mass function is surprisingly similar in shape to the stellar IMF but scaled to a higher mass by a factor of about 3 as can be seen in Figure 2 (Alves et al. 2007). Most of the cores appear to be pressure confined, gravitationally unbound entities whose fundamental physical properties are determined by only a few factors, which include self-gravity, gas temperature, and the simple requirement of pressure equilibrium with the surrounding environment. The entire core population in the Pipe is found to be characterized by a single critical Bonnor-Ebert mass of approximately $2 \mathrm{M}_{\odot}$. This mass coincides with the characteristic mass of the Pipe CMF suggesting that the CMF (and ultimately the stellar IMF) has its origin in the physical process of thermal fragmentation in a pressurized medium.

\section{References}

Alves, J., Lada, C. J., Lada, E. A., Kenyon, S. J. \& Phelps, R. 1998, ApJ, 506, 292

Alves, J., Lombardi, M. \& Lada, C. J. 2007, A\&A, 462, L17

Alves, J. F., Lada, C. J. \& Lada, E. A. 2001, Nat, 409, 159

Froebrich, D. \& Rowles, J. 2010, MNRAS, 406, 1350

Goodman, A. A., Pineda, J. E. \& Schnee, S. L. 2009, ApJ, 692, 91

Kainulainen, J., Beuther, H., Henning, T. \& Plume, R. 2009, A\&A, 508, L35

Lada, C. J., Lada, E. A., Clemens, D. P. \& Bally, J. 1994, ApJ, 429, 694

Lada, C. J., Lombardi, M. \& Alves, J. F. 2009, ApJ, 703, 52

-. 2010, ArXiv e-prints

Lombardi, M. 2009, A\&A, 493, 735

Lombardi, M. \& Alves, J. 2001, A\&A, 377, 1023

Lombardi, M., Alves, J. \& Lada, C. J. 2010a, A\&A, 519, L7

Lombardi, M., Lada, C. J. \& Alves, J. 2008, A\&A, 489, 143

-. 2010b, A\&A, 512, 67

Muench, A. A., Lada, E. A., Lada, C. J. \& Alves, J. 2002, ApJ, 573, 366

Myers, P. C. \& Benson, P. J. 1983, ApJ, 266, 309

Román-Zúñiga, C. G., Alves, J. F., Lada, C. J. \& Lombardi, M. 2010, ArXiv e-prints

Tassis, K., Christie, D. A., Urban, A., Pineda, J. L., Mouschovias, T. C., Yorke, H. W. \& Martel, H. 2010, MNRAS, 408, 1089

Wu, J., Evans, II, N. J., Gao, Y., Solomon, P. M., Shirley, Y. L. \& Vanden Bout, P. A. 2005, ApJL, 635, L173 